\title{
Quasiparticle resonant states induced by a unitary impurity in a $d$-wave superconductor
}

\author{
Jian-Xin Zhu \\ Texas Center for Superconductivity and Department of Physics, University of Houston, Houston, Texas 77204 \\ T. K. Lee \\ Institute of Physics, Academia Sinica, Nankang, Taipei 11529, Taiwan, Republic of China \\ and National Center for Theoretical Sciences, P.O. Box 2-131 Hsinchu, Taiwan 300, Republic of China \\ C. S. Ting \\ Texas Center for Superconductivity and Department of Physics, University of Houston, Houston, Texas 77204 \\ and National Center for Theoretical Sciences, P.O. Box 2-131, Hsinchu, Taiwan 300, Republic of China \\ Chia-Ren $\mathrm{Hu}$ \\ Department of Physics, Texas A\&M University, College Station, Texas 77843
}

(Received 14 September 1999)

\begin{abstract}
The quasiparticle resonant states around a single nonmagnetic impurity with unitary scattering in a $d$-wave superconductor is studied by solving the Bogoliubov-de Gennes equations based on a $t$ - $J$ model. Both the spatial variation of the order parameter and the local density of states (LDOS) around the impurity have been investigated. We find that (i) a particle-hole symmetric system has a single symmetric zero-energy peak in the LDOS regardless of the size of the superconducting coherence length $\xi_{0}$; (ii) for the particle-hole asymmetric case, an asymmetric splitting of the zero-energy peak is intrinsic to a system with a small value of $k_{F} \xi_{0}$.
\end{abstract}

It is now well established ${ }^{1}$ that high- $T_{c}$ superconductors

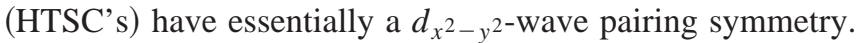
In conventional $s$-wave superconductors, nonmagnetic impurities affect neither the transition temperature nor the superfluid density as dictated by the Anderson theorem. ${ }^{2}$ But in a $d$-wave superconductor (DWSC) with nodes of the energy gap, such impurities can cause a strong pair-breaking effect. ${ }^{3}$ Recently, the local electronic properties in the immediate vicinity of an isolated nonmagnetic impurity in a DWSC has become the topic of increased investigation, ${ }^{4-16}$ as these properties may provide a distinctive signature for the pairing symmetry. It has been theoretically predicted by Balatsky, Salkola and co-workers ${ }^{7,8}$ that, in a DWSC, a single nonmagnetic impurity can generate quasiparticle resonant states at subgap energies. They showed that, for a moderately strong impurity, an asymmetry of the resonance peak near the Fermi energy is induced by the fact that the impurity locally breaks the particle-hole symmetry. However, their theory says that increasing the impurity strength pushes the resonance peak toward the Fermi level, so that, in the unitary limit, the resonance occurs right on the Fermi level, and only a single symmetric zero-energy peak (ZEP) occurs in the local density of states (LDOS) near the impurity. It has also been shown by a finite-size diagonalization ${ }^{10}$ that, in the unitary limit, the lowest eigenvalues are essentially zero, indicative of the appearance of zero-energy states (ZES's). Note that, in Ref. 10 , the chemical potential $\mu$ was taken to be at the center of the tight-binding energy band (i.e., $\mu=0$ ), so that the system has a particle-hole symmetry. This symmetry is also upheld in the continuum-theory treatment of impurities $^{7,8}$ where the self-consistent $t$-matrix approximation is employed. A question which arises naturally is whether, in the unitary limit, the "ZEP' in the LDOS due to the "ZES's', has an asymmetric splitting or not, when particle-hole symmetry is broken in the system. Recently, Tanaka, Kuboki, and Sigrist ${ }^{15}$ concluded with their numerical study that such a splitting is still present, whereas Tsuchiura et al. ${ }^{16}$ made an opposite conclusion in their numerical study, and asserted that the system studied by Tanaka, Kuboki, and Sigrist was too small for their results to be reliable. Experimentally, an asymmetric splitting is clearly observed by Yazdani et al., ${ }^{12}$ whereas Hudson et al. ${ }^{13}$ observed only an off-centered peak with no indication of a splitting. Thus it appears important to settle the issue of whether a unitary nonmagnetic impurity in a pure DWSC can indeed give rise to such an asymmetric splitting in the ' 'ZEP,' as it will decide whether experimental observation of this feature in HTSC's necessarily implies that these SC's do not have pure $d$-wave symmetry, or that the impurity is not in the unitary limit (in which case the asymmetry is tied to the sign of the impurity potential, which may well be a misleading conclusion).

Based on a $t-J$ model, this paper presents an extensive study on the electronic states around a unitary single-site impurity in a DWSC. The spatial variation of the superconducting order parameter (OP) near the impurity, including an induced $s$-wave component, is determined self-consistently. By investigating the sensitivity of the LDOS on both $\mu$ and $\xi_{0}$, we find: (i) when $\mu=0$, so that the system is particlehole symmetric, a single ZEP occurs in the LDOS spectrum which is symmetric with respect to zero energy, regardless of the size of $\xi_{0}$; (ii) as the particle-hole symmetry is broken by letting $\mu \neq 0$, a critical value $\gamma_{c}$ exists, which is larger for larger $|\mu|$, so that for $\gamma \equiv k_{F} \xi_{0}<\gamma_{c}$ the ' ZEP', exhibits an asymmetric splitting. (Here $k_{F}$ is the Fermi wave vector.) ${ }^{17}$ Thus we find that for a particle-hole asymmetric system, a 
sufficiently small coherence length can cause the "ZEP'" to exhibit an asymmetric splitting. Treating such a system by the self-consistent $t$-matrix approximation, which restores the particle-hole symmetry, will then lose this feature and be misleading in this respect.

We consider a $t-J$ model Hamiltonian defined on a twodimensional square lattice:

$$
\begin{aligned}
\mathcal{H}= & -t \sum_{\langle\mathbf{i j}\rangle \sigma} c_{\mathbf{i} \sigma}^{\dagger} c_{\mathbf{j} \sigma}+\sum_{\mathbf{i}} U_{\mathbf{i}} n_{\mathbf{i}}-\mu \sum_{\mathbf{i}} n_{\mathbf{i}} \\
& +\frac{J}{2} \sum_{\langle\mathbf{i} \mathbf{j}\rangle}\left[\mathbf{S}_{\mathbf{i}} \cdot \mathbf{S}_{\mathbf{j}}-\frac{1}{4} n_{\mathbf{i}} n_{\mathbf{j}}\right]+\frac{W}{2} \sum_{\langle\mathbf{i j}\rangle} n_{\mathbf{i}} n_{\mathbf{j}},
\end{aligned}
$$

where the Hilbert space is made of empty and singlyoccupied sites only; summing over $\langle\mathbf{i j}\rangle$ means summing over nearest-neighbor sites; $n_{\mathbf{i}}=\Sigma_{\sigma} c_{\mathbf{i} \sigma}^{\dagger} c_{\mathbf{i} \sigma}$ is the electron number operator on site $\mathbf{i} ; \mathbf{S}_{\mathbf{i}}$ is the spin- $\frac{1}{2}$ operator on site $\mathbf{i}$; and $J$ $>0$ gives the antiferromagnetic superexchange interaction. As in Ref. 18, we have also included a direct nearestneighbor interaction term. $W=0$ and $J / 4$ correspond to two versions of the standard $t-J$ model. This term is introduced to adjust the magnitude of the resultant $d$-wave OP. The scattering potential from the single-site impurity is modeled by $U_{\mathbf{i}}=U_{0} \delta_{\mathrm{i} I}$ with $I$ the index for the impurity site. The slaveboson method ${ }^{19}$ is employed to write the electron operator as $c_{\mathbf{i} \sigma}=b_{\mathbf{i}}^{\dagger} f_{\mathbf{i} \sigma}$, where $f_{\mathbf{i} \sigma}$ and $b_{\mathbf{i}}$ are the operators for a spinon (a neutral spin- $\frac{1}{2}$ fermion) and a holon (a spinless charged boson). Due to the holon Bose condensation at low temperatures, the quasiparticles are determined by the spinon degree of freedom only. Within the mean-field approximation, the Bogoliubov-de Gennes (BdG) equations are derived to be

$$
\sum_{\mathbf{j}}\left(\begin{array}{cc}
H_{\mathrm{ij}} & \Delta_{\mathbf{i j}} \\
\Delta_{\mathbf{i j}}^{\dagger} & -H_{\mathbf{i j}}
\end{array}\right)\left(\begin{array}{c}
u_{\mathbf{j}}^{n} \\
v_{\mathbf{j}}^{n}
\end{array}\right)=E_{n}\left(\begin{array}{c}
u_{\mathbf{i}}^{n} \\
v_{\mathbf{i}}^{n}
\end{array}\right)
$$

with

$$
H_{\mathrm{ij}}=-\left[t \delta+\left(\frac{J}{2}+W\right) \chi_{\mathbf{i j}}\right] \delta_{\mathbf{i}+\delta, \mathbf{j}}+\left(U_{\mathbf{i}}-\mu\right) \delta_{\mathbf{i j}} .
$$

Here $u_{\mathbf{i}}^{n}$ and $v_{\mathbf{i}}^{n}$ are the Bogoliubov amplitudes corresponding to the eigenvalue $E_{n} ; \delta$ and $\chi_{\mathrm{ij}}$ are the doping rate and the bond OP, respectively; and $\delta$ are the unit vectors $\pm \hat{\mathbf{x}}$, $\pm \hat{\mathbf{y}}$. The resonant-valence-bond (RVB) OP $\Delta_{\mathrm{ij}}, \chi_{\mathrm{ij}}$, and $\delta$ are determined self-consistently:

$$
\begin{gathered}
\Delta_{\mathbf{i j}}=\frac{J-W}{2} \sum_{n}\left[u_{\mathbf{i}}^{n} v_{\mathbf{j}}^{n *}+u_{\mathbf{j}}^{n} v_{\mathbf{i}}^{n *}\right] \tanh \left(E_{n} / 2 k_{B} T\right) \delta_{\mathbf{i}+\boldsymbol{\delta}, \mathbf{j}} \\
\chi_{\mathbf{i j}}=\sum_{n}\left\{u_{\mathbf{i}}^{n *} u_{\mathbf{j}}^{n} f\left(E_{n}\right)+v_{\mathbf{i}}^{n} v_{\mathbf{j}}^{n *}\left[1-f\left(E_{n}\right)\right]\right\}
\end{gathered}
$$

and

$$
\delta=1-\frac{2}{N_{a}} \sum_{\mathbf{i}, n}\left\{\left|u_{\mathbf{i}}^{n}\right|^{2} f\left(E_{n}\right)+\left|v_{\mathbf{i}}^{n}\right|^{2}\left[1-f\left(E_{n}\right)\right]\right\},
$$

where $k_{B}$ is the Boltzmann constant; $f(E)=\left[\exp \left(E / k_{B} T\right)\right.$ $+1]^{-1}$ is the Fermi distribution function; and $N_{a}=N_{x} \times N_{y}$ is the number of lattice sites. The BdG equations are solved fully self-consistently for the bulk state first. We then fix the values of $\delta$ and $\chi$ and solve the BdG equations in the presence of a single impurity with the self-consistent $d$-wave RVB OP. The thermally broadened local density of states (LDOS) is then evaluated according to

$$
\rho_{\mathbf{i}}(E)=-2 \sum_{n}\left[\left|u_{\mathbf{i}}^{n}\right|^{2} f^{\prime}\left(E_{n}-E\right)+\left|v_{\mathbf{i}}^{n}\right|^{2} f^{\prime}\left(E_{n}+E\right)\right],
$$

where a factor 2 arises from the spin sum, and $f^{\prime}(E)$ $\equiv d f(E) / d E$. The LDOS $\rho_{\mathrm{i}}(E)$ is proportional to the local differential tunneling conductance which can be measured in a scanning tunneling microscope/spectroscopy (STM/S) experiment. $^{21}$

In the numerical calculation, we construct a superlattice with the square lattice $N_{x} \times N_{y}$ as a unit supercell. As detailed in Ref. 20, this method can provide the required energy resolution for the possible resonant states. Throughout the work, we take the size of the unit supercell $N_{a}=35$ $\times 35$, the number of supercells $N_{c}=6 \times 6$, the temperature $T=0.01 \mathrm{~J}$, and the single impurity potential in the unitary limit $U_{0}=100 \mathrm{~J}$. The values of the other parameters $-\mu, W$, and $t$, are varied in order to investigate the electronic states around a single impurity for various ways to bring about particle-hole asymmetry. The obtained spatial variation of the $d$-wave and the induced extended-s-wave OP components around the impurity, which are defined as $\Delta_{d}(\mathbf{i})$ $=\frac{1}{4}\left[\Delta_{\hat{x}}(\mathbf{i})+\Delta_{-\hat{x}}(\mathbf{i})-\Delta_{\hat{y}}(\mathbf{i})-\Delta_{-\hat{y}}(\mathbf{i})\right]$, and $\Delta_{s}(\mathbf{i})=\frac{1}{4}\left[\Delta_{\hat{x}}(\mathbf{i})\right.$ $\left.+\Delta_{-\hat{x}}(\mathbf{i})+\Delta_{\hat{y}}(\mathbf{i})+\Delta_{-\hat{y}}(\mathbf{i})\right]$, is similar to Fig. 1 of Ref. 9. These OP components have the following characteristics: The $d$-wave component decreases continuously to zero from its bulk value as the impurity site is approached, in the scale of the coherence length $\xi_{0} \equiv \hbar v_{F} / \pi \Delta_{\text {max }}$, with the depleted region extending farther in the nodal directions if $\xi_{0}$ is larger. Here $\Delta_{\max }=4 \Delta_{0}$ with $\Delta_{0}$ the bulk value of the $d$-wave OP defined in the real space on a nearest-neighbor bond, and $v_{F}$ is the Fermi velocity. The $s$-wave component is zero at the impurity site and also at infinity. It has line-nodes along the $\{110\}$ and $\{1 \overline{1} 0\}$ directions, and changes sign across any nodal line. Unlike the pairing state at a $\{110\}$ surface of a DWSC, which can break the time-reversal symmetry, the pairing state near a single impurity conserves time-reversal symmetry. This difference can be understood from the Ginzburg-Landau (GL) theory, ${ }^{22}$ in that a mixed gradient term favors the $d$ - and induced $s$-wave OP components to be in phase, but it vanishes near a $\{110\}$ surface, whence the fourth order $s-d$ coupling term can establish an $s+i d$ pairing state.

Figure 1 shows the LDOS as a function of energy on sites one and two lattice spacings along the (100) direction from the impurity and on the corner site of the unit supercell. The values of the parameters are labeled on the figure. Note that the LDOS at the corner site has recovered the bulk DOS, by exhibiting a gaplike feature with the gap edges at $\pm \Delta_{\max }$. This resemblance indicates that the unit-cell size and the number of unit cells are large enough for uncovering the physics intrinsic to an isolated impurity. As shown in Fig. 1, we find that the LDOS spectrum near the impurity is highly sensitive to the position of $\mu$ within the energy band, and the parameter $\gamma$. In Fig. 1(a), $\mu=0$ (Ref. 23) and $\gamma=0.80$, a single ZEP occurs in the LDOS on the nearest-neighbor site 


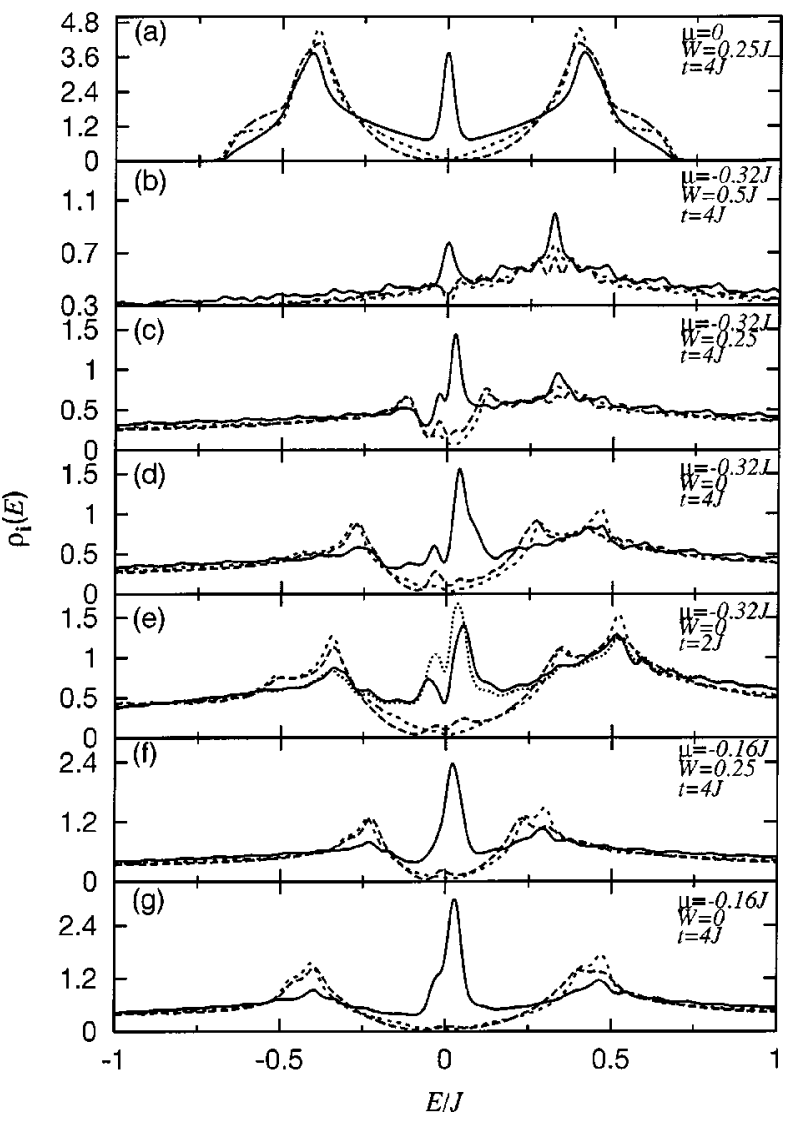

FIG. 1. Local density of states as a function of energy on sites one (solid line) and two lattice (dashed line) spacings along the (100) direction away from the impurity, and on the corner site (short-dashed line) of the unit cell. The parameter values have been correspondingly labeled on each panel. Also shown in the (e) panel is the local density of states on the site nearest neighbor to the impurity obtained with a pure bulk $d$-wave order parameter (dotted line).

of the impurity, similar to the prediction of the continuum theory ${ }^{7,8}$ and the eigenvalue calculation in Ref. 10 . In addition, as a reflection of the particle-hole symmetry, the whole LDOS spectrum is symmetric about $E=0$. We have also studied the cases (not shown) with the same $\mu=0$ and $t$ $=4 \mathrm{~J}$ but with $W=0$ and $W=0.5 \mathrm{~J}$ (corresponding to $\gamma$ $=0.27$ and 2.0), and found that the above feature remains unchanged, which allows us to conclude that as long as the system is particle-hole symmetric, only a single symmetric ZEP exists for all $\gamma$. When $\mu$ is not zero, the system is particle-hole asymmetric, and the LDOS spectrum becomes asymmetric. [see Figs. 1(b) $-1(\mathrm{~g})$ ]. In Figs. 1(b)-1(e), $\mu=-0.32 J$ is fixed, and $W$ and $t$ are varied in order to change $\gamma$. For a large $\gamma=91.7$, we see a single ZEP in the LDOS [see Fig. 1(b)]. When $\gamma$ is lowered to 16.5, the "ZEP' begins to evolve into a double-peaked structure with the $E>0$ peak having the dominant spectral weight over the $E<0$ peak. For a further decreased $\gamma=6.05$, the spectral weight of the peak at $E<0$ is enhanced (see Fig. 1(d)). As seen in Fig. 1(e), this enhancement becomes even more pronounced when $\mu$ is made close to the edge of a very narrow energy band so that $\gamma$ becomes as small as 2.85. When $\mu$ $=-0.16 \mathrm{~J}$, we only observe a single ZEP although $\gamma$ is as small as 5.7 [for Fig. 1(f)] and 2.9 [for Fig. 1(g)], except that

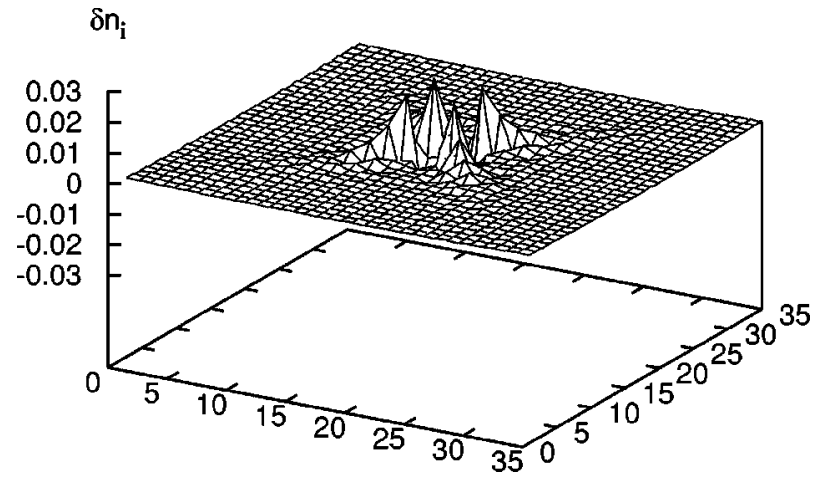

FIG. 2. Spatial variation of the charge distribution around the impurity with the parameter values given in Fig. 1(e).

a tendency of the splitting can be identified in the latter case. This tendency of the splitting has been observed clearly in STM tunneling spectroscopy measurements [see Fig. 4(A) of Ref. 12]. It should be emphasized that the ZEP splitting obtained here has a different origin from that found by Tanaka, Kuboki, and Sigrist. ${ }^{15}$ We have re-examined their results by choosing the same parameter values and the system size $(18 \times 18)$. When the LDOS spectrum is displayed in a wide energy landscape, many split DOS peaks appear with no well-defined gaplike feature identifiable. But as the system size is enlarged by the supercell technique, the calculation only shows a single ZEP in the LDOS, which indicates that the splitting of ZEP obtained in Ref. 15 is indeed due to the size effect. On the other hand, we have also calculated the excess charge distribution due to the presence of the impurity $\left(\propto \delta n_{\mathbf{i}}=\left\langle n_{\mathbf{i}}\right\rangle-n_{0}\right.$, where $n_{0}$ is the average particle occupation on each site for the bulk system). We find that this distribution is anisotropic, with its magnitude having tails extending along the nodal directions (see Fig. 2). Because Fig. 2 is obtained with the parameter values given in Fig. 1(e) which gives a small $\gamma(=2.85)$ value, the exhibited tail is short. A similar calculation with the model parameters given in Fig. 1(f) (not shown) shows that the charge distribution is similar to that displayed in Fig. 2 except for a longer tail along the nodal directions due to the larger $\gamma$ $(=5.7)$. This similarity in the charge distributions for a split and an unsplit ZEP's disproves the assertion made in Ref. 15 that the local charge-density oscillation is the cause of the ZEP splitting. We mention in passing that we have also found that the excess charge density decays exponentially along the nodal directions instead of the $r^{-2}$ dependence from the impurity. But we do not think that this finding invalidates the assertion in Ref. 7 that the wave function of the impurity resonant state has a $1 / r$ decay along the nodal directions, which can lead to a long-range interaction between the impurities. However, we do believe that since we have obtained essentially the bulk density of states in several neighboring points near the corner of the supercell, the interaction between the neighboring impurities should be negligible in the cell size we have chosen to work with. Thus we believe that it is very unlikely that the splitting of the ZEP we obtain is due to this interaction. Since the $s$-wave OP component induced near the impurity is in phase with the dominant $d$-wave component, the splitting of the ZEP we found is not due to a local broken time-reversal symmetry. Finally, as shown in Fig. 1(e), the splitting is also exhibited 
in a non-self-consistent calculation with a spatially uniform bulk $d$-wave OP, showing that the suppression of the $d$-wave OP component, and the induction of the s-wave component, have little to do with the splitting. All of these points lead us to the conclusion that, for the particle-hole asymmetric case, the splitting of the ZEP is intrinsic to the system with a short coherence length, and the critical value $\gamma_{c}$, below which the ZEP is split into an asymmetric double-peak, is simply a reflection that the system has reached a critical extent in its deviation from particle-hole symmetry. We thus propose to understand these results qualitatively as follows: The "ZES's" induced by a unitary non-magnetic impurity have essentially the same physical origin as the "midgap states" predicted to exist on the surfaces/interfaces of a DWSC. ${ }^{24}$ Their existence is implied topologically by the Atiyah-Singer index theorem, ${ }^{25}$ which applies to particle-hole-symmetric Dirac-like operators. When this symmetry is mildly broken the midgap states are expected to still exist but no longer exactly "midgap." The BdG equations become Dirac-like equations only under the WKBJ approximation (which is a part of the self-consistent $t$-matrix approximation), the error of which is measured by the parameters $|\mu|$ and $\gamma^{-1}$. For $\mu=0$, the system has exact particle-hole symmetry for all $\gamma$. Thus smaller $|\mu|$ should imply smaller $\gamma$ needed to reach the same deviation from particle-hole symmetry, hence a smaller $\gamma_{c}$ below which an asymmetric splitting of the ZEP appears.

In summary, we have presented an extensive study on the quasiparticle resonant states induced by a unitary nonmagnetic impurity in a DWSC. The results have clarified some conflicting conclusions in the literature, and should be of value for the proper analysis of the STM/S results obtained on HTSC's around an isolated impurity.

We are grateful to M. Salkola, M. E. Flatté, and A. Yazdani for valuable discussions. This work was supported by the Texas Center for Superconductivity at the University of Houston, the Robert A. Welch Foundation, and the Texas Higher Education Coordinating Board. Free computing time from the Texas A\&M Supercomputer Center is also gratefully acknowledged.
${ }^{1}$ D.J. Scalapino, Phys. Rep. 250, 329 (1995); D.J. van Harlingen, Rev. Mod. Phys. 67, 515 (1995).

${ }^{2}$ P.W. Anderson, J. Phys. Chem. Solids 11, 26 (1959).

${ }^{3}$ L.P. Gorkov, Pis'ma Zh. Éksp. Teor. Fiz. 40, 351 (1984) [JETP Lett. 40, 1155 (1984)]; K. Ueda and T. M. Rice, in Theory of Heavy Fermions and Valence Fluctuations, edited by T. Kasuya and T. Saso (Springer, Berlin, 1985).

${ }^{4}$ P.A. Lee, Phys. Rev. Lett. 71, 1887 (1993).

${ }^{5}$ J.M. Byers, M.E. Flatté, and D.J. Scalapino, Phys. Rev. Lett. 71, 3363 (1993).

${ }^{6}$ C.H. Choi, Phys. Rev. B 50, 3491 (1994).

${ }^{7}$ A.V. Balatsky, M.I. Salkola, and A. Rosengren, Phys. Rev. B 51, 15547 (1995); A.V. Balatsky and M.I. Salkola, Phys. Rev. Lett. 76, 2386 (1996).

${ }^{8}$ M.I. Salkola, A.V. Balatsky, and D.J. Scalapino, Phys. Rev. Lett. 77, 1841 (1996); M.I. Salkola, A.V. Balatsky, and J.R. Schrieffer, Phys. Rev. B 55, 12648 (1997).

${ }^{9}$ M. Franz, C. Kallin, and A.J. Berlinsky, Phys. Rev. B 54, R6897 (1996).

${ }^{10}$ Y. Onishi et al., J. Phys. Soc. Jpn. 65, 675 (1996).

${ }^{11}$ M.E. Flatté and J.M. Byers, Phys. Rev. Lett. 80, 4546 (1998).

${ }^{12}$ A. Yazdani et al., Phys. Rev. Lett. 83, 176 (1999).

${ }^{13}$ E.W. Hudson et al., Science 258, 88 (1999).

${ }^{14}$ A. Shnirman et al., Phys. Rev. B 64, 7517 (1999).

${ }^{15}$ H. Tanaka, K. Kuboki, and M. Sigrist, Int. J. Mod. Phys. B 12, 2447 (1998).
${ }^{16}$ H. Tsuchiura et al., J. Phys. Soc. Jpn. 68, 2510 (1999).

${ }^{17}$ That impurity-induced resonances may not lie exactly midgap in the absence of particle-hole (or band) symmetry is not new, as has been pedagogically reviewed by R. Joynt [J. Low Temp. Phys. 109, 811 (1997)], but we believe that the importance of the parameter $\gamma$, and the possible existence of a crossover behavior at a critical value $\gamma_{c}$ have not been reported previously.

${ }^{18}$ R. Micnas, J. Ranninger, and S. Robaszkiewicz, Rev. Mod. Phys. 62, 113 (1990).

${ }^{19}$ G. Kotliar and J. Liu, Phys. Rev. B 38, 5142 (1988), and references therein.

${ }^{20}$ J.-X. Zhu, B. Friedman, and C.S. Ting, Phys. Rev. B 59, 3353 (1999).

${ }^{21}$ M. Tinkham, Introduction to Superconductivity (McGraw-Hill, New York, 1975).

${ }^{22}$ J.H. Xu, Y. Ren, and C.S. Ting, Phys. Rev. B 52, 7663 (1995).

${ }^{23}$ Notice that at small doping, in particular at zero doping, the mean-field slave-boson approach adopted here is not representative of the physics of the $t-J$ model at all, but just a tool for analyzing the resonant states in the particle-hole symmetric limit.

${ }^{24}$ C.-R. Hu, Phys. Rev. Lett. 72, 1526 (1994); Phys. Rev. B 57, 1266 (1998)

${ }^{25}$ M. Nakahara, Geometry, Topology, and Physics (Adam Hilger, New York, 1990), Chap. 12, and especially pp. 420-424. 